\title{
Circumstellar emission in Be/X-ray binaries of the Magellanic Clouds and the Milky Way ${ }^{\star}$
}

\author{
M. S. Riquelme ${ }^{1}$, J. M. Torrejón ${ }^{1,2}$, and I. Negueruela ${ }^{1}$ \\ ${ }^{1}$ Departamento de Física, Ingeniería de Sistemas y Teoría de la Señal, Escuela Politécnica Superior, Universidad de Alicante, Spain \\ e-mail: mriquelme@dfists.ua.es \\ ${ }^{2}$ Instituto de Física Aplicada a las Ciencias y las Tecnologías, Universidad de Alicante, 03690 Alicante, Spain
}

Received 21 July 2011 / Accepted 20 December 2011

\begin{abstract}
Aims. We study the optical and near-infrared colour excesses produced by circumstellar emission in a sample of Be/X-ray binaries. Our main goals are exploring whether previously published relations, valid for isolated Be stars, are applicable to Be/X-ray binaries and computing the distance to these systems after correcting for the effects of the circumstellar contamination.

Methods. Simultaneous $U B V R I$ photometry and spectra in the 3500-7000 ̊ spectral range were obtained for 11 optical counterparts to Be/X-ray binaries in the LMC, 5 in the SMC and 12 in the Milky Way. As a measure of the amount of circumstellar emission we used the $\mathrm{H}_{\alpha}$ equivalent width corrected for photospheric absorption.

Results. We find a linear relationship between the strength of the $\mathrm{H}_{\alpha}$ emission line and the component of $E(B-V)$ originating from the circumstellar disk. This relationship is valid for stars with emission lines weaker than $E W \approx-15 \AA$. Beyond this point, the circumstellar contribution to $E(B-V)$ saturates at a value $\approx 0.17$ mag. A similar relationship is found for the $(V-I)$ near infrared colour excess, albeit with a steeper slope and saturation level. The circumstellar excess in $(B-V)$ is found to be about five times higher for Be/X-ray binaries than for isolated Be stars with the same equivalent width $E W\left(\mathrm{H}_{\alpha}\right)$, implying significant differences in the physical properties of their circumstellar envelopes. The distance to Be/X-ray binaries (with non-shell Be star companions) can only be correctly estimated by taking into account the excess emission in the $V$ band produced by free-free and free-bound transitions in the circumstellar envelope. We provide a simple method to determine the distances that includes this effect.
\end{abstract}

Key words. circumstellar matter - X-rays: binaries - stars: early-type

\section{Introduction}

$\mathrm{Be} / \mathrm{X}$-ray binaries comprise the major subclass within the high mass X-ray binaries, and the majority of X-ray pulsars belong to this class (see Reig 2011, for a comprehensive recent review). They are composed of a neutron star (NS) that accretes circumstellar material from the disk around a massive Be star. Be stars in X-ray binaries have spectral types restricted to a fairly narrow interval, O9 to B3 and luminosity classes V to III (Negueruela 1998). These stars are surrounded by a circumstellar envelope that profoundly alters the emerging spectrum. Spectra of Be stars show some essential characteristics. 1) The Balmer lines are affected by the emission that is produced by bound-bound transitions in the envelope, which fills in the photospheric lines or drives them into emission. 2) Free-free transitions in the envelope (thermal Bremsstrahlung) produce an excess of photons at long wavelengths, compared to a B star of the same spectral type. This effect is referred to as infrared excess and increases with wavelength. 3) Thermal Bremsstrahlung also makes the star appear brighter, compared to a B star of the same luminosity class. Other mechanisms can help to modify even further the brightness of the system. Thomson scattering in the envelope, for example, could add photons into the observer's line of sight, which would additionally increase the brightness of the star.

\footnotetext{
* Partially based on observations collected at the European Southern Observatory, La Silla, Chile (66.D-0292; 074.D-0529) and the Nordic Optical Telescope.
}

Owing to these effects, the direct application of the photometric and spectroscopic techniques for calculating the physical parameters of a star leads to incorrect results. In particular, the distances to some Be/X-ray binaries, and therefore their X-ray luminosities, could be underestimated, as we will see. All these effects are directly connected with the envelope and, therefore, a relationship between them and the overall amount of emission is expected.

In this paper, we present a method to correctly treat these effects by means of simple correlations between the circumstellar excess in $(B-V)$ and $(V-I)$ colours and the amount of emission, as measured by the $\mathrm{H}_{\alpha}$ equivalent width. To this goal, we have observed a sample of Be/X-ray binaries in the Magellanic Clouds (LMC and SMC) and in the Milky Way galaxy (MWG). Such relations had been established so far for isolated Be stars, that is to say, Be stars not known to be in binaries (Dachs et al. 1988, for bright field stars, Fabregat \& Torrejón 1998, for Be stars in open clusters). Nevertheless, we now know that the circumstellar envelope of Be stars in binaries is substantially modified by the presence of the accreting compact object. Circumstellar envelopes in binary systems tend to be geometrically more compact and optically thicker (Reig et al. 1997; Okazaki \& Negueruela 2001). It has been shown in the past years by direct interferometric results that the disk is indeed truncated in all cases studied (Tycner et al. 2006; Gies et al. 2007; Gründstrom \& Gies 2006). There is evidence that the circumstellar reddening given by the calibrations for isolated 
Be stars is too small when applied to Be/X-ray binaries for a given amount of emission (Zamanov et al. 2001). Therefore, the question arises whether these relationships can be safely applied as well to $\mathrm{Be} / \mathrm{X}$-ray binaries. In this paper we aim to answer this question.

\section{Observations}

\subsection{Sample selection and instrumentation}

In order to be able to decouple the interstellar reddening and extinction from the circumstellar reddening and brightening ${ }^{1}$ we need a set of programme stars with well-known distances and interstellar reddenings as well as good determinations of the spectral types. In principle we could choose Be/X-ray binaries in well-studied open clusters and follow an approach similar to the one used by Fabregat \& Torrejón (1998). Unfortunately, such stars do not seem to exist or are very scarce. Therefore we used stars in the Large Magellanic Cloud (LMC) whose distance and reddening are satisfactorily studied by independent methods. Furthermore, the LMC presents a population distribution that is similar to the Milky Way Galaxy (MWG; Antoniou et al. 2009) as well as a similar metallicity. The sample is almost complete because we have observed 11 out of $15 \mathrm{BeX}$ known in the LMC. As a control sample we included five BeX in the Small Magellanic Cloud (SMC), chosen randomly amongst those with already determined and published spectral types. Finally we have chosen a sample of BeX in the MWG covering a wide range of emission strengths and spectral types. All 28 observed stars have spectral types already determined and published (the references are given in Table 4). This is mandatory, because the parameters of the underlying B star will be used as input for the calibration.

Because Be stars are variable even on timescales of hours, we obtained simultaneous UBVRI Bessel photometry and Balmer spectroscopy in three different runs using the following telescopes and instrumentation:

- The Danish Faint Object Spectrograph and Camera (DFOSC), attached to the Danish $1.54 \mathrm{~m}$ telescope, located at the ESO site in La Silla (Chile) during a run on 2001 February, 8-10.

- The Andalucian Faint Object Spectrograph and Camera (ALFOSC), installed on the $2.5 \mathrm{~m}$ Nordic Optical Telescope (NOT) in La Palma (Spain) during a run on 2001 July, 2-5.

- The ESO Multi-Mode Instrument (EMMI), at the $3.6 \mathrm{~m} \mathrm{New}$ Technology Telescope (NTT), also located in La Silla, on 2004 December, $14 \& 15$.

These instruments allow the sequential acquisition of images and spectra, with virtually no time delay, which is mandatory for reducing the uncertainty produced by short-term variability.

\subsection{Photometry}

The photometric images were taken sequentially with the Bessell $U B V R I$ (broadband filters). In some runs only $B V R$ photometry is available.

For data reduction we used the Starlink suite ${ }^{2}$. Bias subtraction and flatfielding were carried out on all frames using the CCDPACK package (Draper et al. 2000). Photometry was performed using the PSF fitting optimum extraction method implemented in GAIA (Draper \& Gray 2000).

\footnotetext{
${ }^{1}$ For classical Be stars. See discussion below for Be shell stars.

2 Now available at http://starlink.jach.hawaii.edu/
}

Calibration of our instrumental magnitudes was carefully determined from eight different standards fields, taken from the list of Landolt (1995): Lan 95, Rubin 152 and PG0942 for the first run, Lan 107-602, Lan 110-230 and Lan 113-163 for the second and Lan 96 and Lan 97 for the third run, respectively. All these fields were followed throughout the night at a wide range of airmasses. The standardization process was carried out using the following set of equations:

$$
\begin{aligned}
& U-u=\xi_{u}+A_{u}(U-B)+K_{u} X+K_{u}^{\prime} X(U-B) \\
& B-b=\xi_{b}+A_{b}(B-V)+K_{b} X+K_{b}^{\prime} X(B-V) \\
& V-v=\xi_{v}+A_{v}(B-V)+K_{v} X+K_{v}^{\prime} X(B-V) \\
& R-r=\xi_{r}+A_{r}(V-R)+K_{r} X+K_{r}^{\prime} X(V-R) \\
& I-i=\xi_{i}+A_{i}(R-I)+K_{i} X+K_{i}^{\prime} X(R-I),
\end{aligned}
$$

where $U$ and $u$ are the standard and instrumental magnitudes, $\xi_{u}$ is the zero point, $K_{u}$ and $K_{u}^{\prime}$ are extinction coefficients, $X$ is the airmass and $A_{u}$ is a constant. Similar notation is used for the remaining filters.

Final calibration equations were applied to all programme stars, resulting in the magnitudes quoted in Table 1. Internal mean errors were judged from the scatter between various measurements of the PSF fitting and photon-counting statistics. These were always less than $0.01 \mathrm{mag}$. After the standardization process, we finally assigned an error to our calibration given by the rms of the residuals between listed and calculated magnitudes for the standard stars. These errors are quoted in Table 2.

\subsection{Spectroscopy}

Following each photometric sequence, medium resolution spectroscopy was performed in the spectral classification and Balmer $\mathrm{H}_{\alpha}$ regions. The details of the several instrumental setups used are given in Table 3. Slits of $1^{\prime \prime}$ were used in all runs. Spectroscopic data were reduced using Starlink packages CCDPACK (Draper et al. 2000) and FIGARo (Shortridge et al. 1997) using standard techniques. After correcting the images for bias and flatfield, spectra were extracted from the object and the sky. This last spectrum was subtracted from that of the object and the resulting spectrum was wavelength-calibrated. For that purpose He-Ar calibration lamp spectra were taken following each programme star observation. Integration times were chosen to obtain signal to noise ratios (S/N) higher than 150 . Finally, several spectrophotometric standard stars were observed each night to allow for absolute flux calibration. In Fig. 1, left panel, we show the spectrum of the programme star KS $1947+300$ as an example. Note the strong $\mathrm{H}_{\alpha}$ emission and reddening.

All spectra were subsequently analysed using the Starlink DIPSo package (Howarth et al. 1998). In order to measure the observed equivalent width of the $\mathrm{H}_{\alpha}$ emission line, we normalized the continuum to unity, as shown in Fig. 1, right panel, which allows for a more precise assessment of the wings extension. All lines showed a full width at half maximum ( $F W H M)$ much higher than the spectral resolution of the different instrumental setups. Therefore, the impact of spectral resolution on the measured $E W$ is negligible.

Errors in the equivalent width measurements were estimated averaging over several adopted continua as well as through several assessments of the wings extension. The process of sky subtraction can introduce non negligible errors around the $\mathrm{H}_{\alpha}$ line because there is often strong and spatially variable diffuse $\mathrm{H}_{\alpha}$ emission in the regions where the $\mathrm{Be} / \mathrm{X}$-ray binaries lie in the Magellanic Clouds. In order to asses and minimize these effects we used a narrow $1^{\prime \prime}$ slit, which minimizes the sky contribution. Next we extracted sky spectra from several positions along 
M. S. Riquelme et al.: Circumstellar emission in Be/X-ray binaries of the Magellanic Clouds and the Milky Way

Table 1. UBVRI photometry for BeX stars in LMC, SMC and the Milky Way are listed in Cols. 3 through 7.

\begin{tabular}{lccccccc}
\hline \hline Galaxy & Star & $U$ & $B$ & $V$ & $R$ & $I$ & Run \\
\hline LMC & CAL E & 12.89 & 14.03 & 14.17 & 14.10 & 14.10 & Danish \\
& CAL 9 & 13.56 & 14.72 & 14.81 & 14.77 & $\ldots$ & Danish \\
& RX J0516.0-6916 & $\ldots$ & 14.99 & 15.12 & 15.15 & $\ldots$ & NTT14 \\
& RX J0516.0-6916 & $\ldots$ & 15.06 & 15.15 & 15.19 & $\ldots$ & NTT15 \\
& RX J0520.5-6932 & 13.31 & 14.46 & 14.46 & 14.40 & 14.23 & Danish \\
& RX J0529.8-6556 & 13.54 & 14.43 & 14.59 & 14.50 & 14.56 & Danish \\
& EXO 0531.1-6609 & $\ldots$ & 14.74 & 14.85 & 14.81 & $\ldots$ & NTT14 \\
& EXO 0531.1-6609 & $\ldots$ & 14.76 & 14.82 & 14.80 & $\ldots$ & NTT15 \\
& RX J0531.5-6518 & $\ldots$ & 15.93 & 15.99 & 16.05 & $\ldots$ & NTT14 \\
& RX J0531.5-6518 & $\ldots$ & 15.89 & 15.99 & 16.01 & $\ldots$ & NTT15 \\
& RX J0535.0-6700 & $\ldots$ & 14.86 & 14.94 & 14.91 & $\ldots$ & NTT14 \\
& 1A 0538-66 & 13.64 & 14.68 & 14.91 & 14.93 & 15.16 & Danish \\
& 1A 0538-66 & $\ldots$ & 15.12 & 15.25 & 15.29 & $\ldots$ & NTT14 \\
& SAX J0544.1-7100 & $\ldots$ & 15.35 & 15.23 & 15.18 & $\ldots$ & NTT14 \\
& H 0544-66 & $\ldots$ & 15.29 & 15.49 & 15.58 & $\ldots$ & NTT14 \\
SMC & RX J0051.9-7311 & $\ldots$ & 14.40 & 14.42 & 14.36 & $\ldots$ & NTT15 \\
& RX J0052.1-7319 & $\ldots$ & 14.86 & 14.86 & 14.80 & $\ldots$ & NTT15 \\
& RX J0059.2-7138 & $\ldots$ & 14.04 & 14.07 & 14.06 & $\ldots$ & NTT15 \\
& RX J0101.0-7206 & $\ldots$ & 15.68 & 15.72 & 15.60 & $\ldots$ & NTT15 \\
& RX J0101.3-7211 & $\ldots$ & 15.47 & 15.53 & 15.54 & $\ldots$ & NTT15 \\
MWG & 4U 0115+634 & 16.96 & 16.96 & 15.54 & $\ldots$ & $\ldots$ & NOT \\
& 3A 0726-260 & 11.32 & 12.12 & 11.61 & 11.12 & 10.88 & Danish \\
& GRO J1008-57 & 17.21 & 16.74 & 15.08 & 13.77 & 12.68 & Danish \\
& LS 1698 & 11.34 & 11.87 & 11.40 & 10.95 & 10.74 & Danish \\
& 1A 1118-61 & 12.63 & 12.95 & 12.09 & 11.26 & 10.69 & Danish \\
& GX 304-1 & 16.46 & 15.63 & 13.94 & 12.69 & 11.67 & Danish \\
& SS 397 & 13.97 & 13.78 & 12.30 & 11.49 & 10.49 & NOT \\
& XTE 1946+274 & 19.05 & 18.63 & 16.89 & 15.65 & 14.78 & NOT \\
& KS 1947+300 & 14.58 & 15.01 & 14.08 & 13.48 & 12.78 & NOT \\
& Cep X-4 & 15.20 & 15.47 & 14.45 & 13.78 & 13.01 & NOT \\
& 4U 2206+54 & 9.27 & 10.01 & 9.67 & 9.59 & 9.39 & NOT \\
& SAX J2239.3+6116 & 16.19 & 16.06 & 14.55 & 13.60 & 12.74 & NOT \\
\hline & & & & & & &
\end{tabular}

Notes. $U$-band and $I$-band photometry was not performed during the NTT campaign.

Table 2. $U B V R I$ photometry errors for the different runs.

\begin{tabular}{lccccc}
\hline \hline$U$ & $B$ & $V$ & $R$ & $I$ & Run \\
\hline 0.03 & 0.02 & 0.02 & 0.02 & 0.03 & Danish \\
$\cdots$ & 0.03 & 0.03 & 0.03 & $\cdots$ & NTT14 \\
$\cdots$ & 0.02 & 0.02 & 0.02 & $\ldots$ & NTT15 \\
0.03 & 0.03 & 0.01 & 0.02 & 0.06 & NOT \\
\hline
\end{tabular}

the slit, including at least two positions close to the target spectrum, and checked for possible variations. The background spectra appeared to be quite stable. Finally, they were averaged and the resulting spectrum subtracted from the that of the star. Final errors were calculated adding the spectrum and model contributions quadratically. The estimated equivalent width errors in the model absorption line is $\pm 0.1 \AA$. The errors in the measured emission line were always lower than $1 \AA$ for the stars in the Clouds and lower than $2 \AA$ for stars in the MWG. Final errors are given in Tables 5 and 8 .

\section{Circumstellar emission and reddening}

\subsection{Colour excess}

As pointed out in the introduction, material surrounding the massive star produces three main effects in the spectrum. In this section we are concerned with the first two. The total excess of photons in the optical is the sum of two components: the circumstellar excess, $E^{\mathrm{cs}}(B-V)$, entirely caused by the envelope, and
Table 3. Grism characteristics for each instrumental setup.

\begin{tabular}{lccccc}
\hline \hline Instrument & $\begin{array}{c}\text { Grism } \\
\#\end{array}$ & $\begin{array}{c}\lambda_{\mathrm{c}} \\
(\AA)\end{array}$ & $\begin{array}{c}\text { Dispersion } \\
(\AA / \mathrm{A} i \mathrm{x})\end{array}$ & $\begin{array}{c}\Delta \lambda \\
(\AA)\end{array}$ & $\begin{array}{c}\text { Range } \\
(\AA)\end{array}$ \\
\hline DFOSC & 3 & 3900 & 2.55 & 5.5 & $3300-6400$ \\
& 5 & 6500 & 3.3 & 7.5 & $5200-10200$ \\
ALFOSC & 3 & 4300 & 2.3 & 6.2 & $3200-6700$ \\
& 5 & 7000 & 3.1 & 8.4 & $5000-10250$ \\
EMMI & 5 & 5300 & 0.83 & 4 & $3800-7020$ \\
\hline
\end{tabular}

Notes. Central wavelengths $\lambda_{\mathrm{c}}$ correspond to the maximum efficiency.

the interstellar excess, $E^{\text {is }}(B-V)$, produced by the interstellar material lying between the system and the observer.

Interstellar material consists mainly of gas and dust. The gas absorbs radiation at very specific wavelengths, leaving absorption lines in the spectrum of the star. The dust, however, scatters the star light over a continuum of wavelenghts. The scatter cross section varies as $\sigma \sim \lambda^{-4}$. Because blue photons are more easily scattered out of the star light beam than the red photons, the spectrum of the star becomes reddened.

On the other hand, the thermal free electrons in the envelope scatter off ions via free-free transitions, thereby emitting a continuum spectrum. This emission spectrum increases its yield towards long wavelengths and is very strong in the infrared. The net effect over the observed spectrum will then be:

$E^{\text {total }}(B-V)=E^{\mathrm{cs}}(B-V)+E^{\mathrm{is}}(B-V)$. 

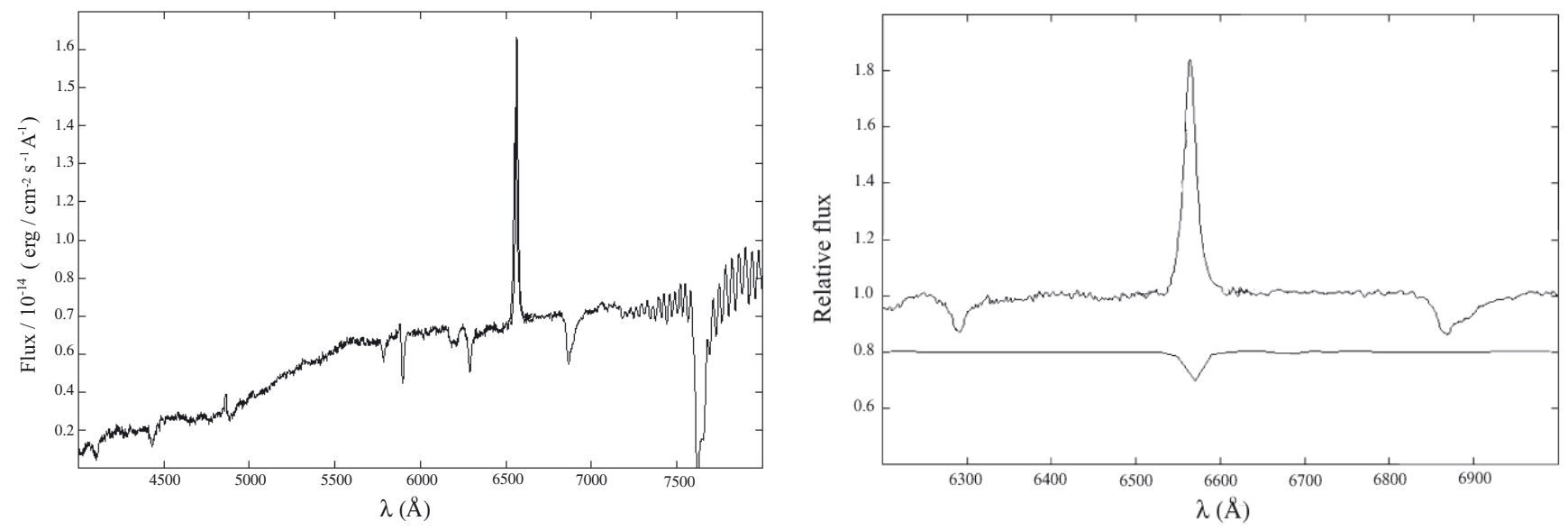

Fig. 1. Left panel: flux-calibrated spectra of KS 1947+300. Note the strong $\mathrm{H}_{\alpha}$ emission line at $6500 \AA$ and the reddening. Right panel: zoom of the emission line normalized to continuum. The Kurucz atmosphere model with the corresponding $\mathrm{H}_{\alpha}$ is also shown with an offset for a better comparison.

The wavelength dependence of these two excesses, or reddenings, is completely different: the interstellar diminishes towards the red, while the circumstellar increases towards the red. But their effects upon the spectrum are indistinguishable. In order to separate these two contributions, we need a set of $\mathrm{Be} / \mathrm{X}$-ray binaries with known distances and reddenings. As explained before, no Be/X-ray binaries in open clusters have been detected so far. Therefore we observed stars in the LMC and the SMC because the interstellar reddening is already sufficiently known a priori by independent methods.

Equation (2) can be explicitly split into two contributions:

$E^{\mathrm{cs}}(B-V)=(B-V)^{*}-(B-V)_{0}$

and

$E^{\mathrm{is}}(B-V)=(B-V)_{\mathrm{obs}}-(B-V)^{*}$,

where $(B-V)^{*}$ is the colour of the star+envelope system (before interstellar reddening). Therefore the total excess, as defined previously in Eq. (2), can be obtained as

$E^{\text {total }}(B-V)=(B-V)_{\mathrm{obs}}-(B-V)_{0}$,

where $(B-V)_{\mathrm{obs}}$ is the observed colour computed from Table 1 and $(B-V)_{0}$ is the intrinsic colour for the corresponding spectral type (Table 4) as given by Schmidt \& Kaler (1982).

For some stars the spectral type is still uncertain. The spectral lines are filled in by emission and this renders the classification quite uncertain in extreme cases. When two spectral types are possible, calculations were made for both, and we chose the value that matched the distance and interstellar reddening of the Clouds correctly. For example, in the case of 1A 0538-66, the luminosity class quoted in the literature is III. However, a class $\mathrm{V}$ fits into the expected value for the distance to the LMC much better. Therefore we chose this second value. Intrinsic $(B-V)$ colours, effective temperatures and $\log g$ were taken from Schmidt \& Kaler (1982). Intrinsic $(V-I)$ colours were taken from Ducati et al. (2001). For consistency, the spectral type deduced from our spectra was compared with the published values whenever possible. A good agreement was found. Owing to the limited spectral resolution of our data we will use the published values. All these parameters are listed in Table 4.

The errors for the $(B-V)_{\text {obs }}$ were calculated adding the contributions of both filters quadratically. This error was considered as the error for the total excess, which is equivalent to assuming that the error in the theoretical values of $(B-V)$ for every spectral type is negligible. Of course this is not strictly correct because we always have an observational error of about \pm one subtype in the spectral type determination. Fortunately, as can be seen in Table 4 , the spectral types are concentrated in a fairly narrow interval, from $\mathrm{B} 0$ to $\mathrm{B} 1$. This covers a range in theoretical colours of about $\Delta(B-V)_{0}=0.03$, which, when added quadratically to the photometric colour errors, yields a final uncertainty of \pm 0.06 .

\section{2. $E^{\text {total }}(B-V)$ vs. $W^{\text {env }}\left(H_{\alpha}\right)$}

Next we compared our spectrum with the stellar atmosphere models of Kurucz (1979), for the effective temperatures and gravities given in Table 4. These models represent the spectrum of the underlying star without reddening and without emission. Then we measured the equivalent widths of the $\mathrm{H}_{\alpha}$ line, both in the observed spectrum, $W^{\mathrm{spc}}\left(\mathrm{H}_{\alpha}\right)$, normally in emission, and in the model, $W^{\mathrm{mdl}}\left(\mathrm{H}_{\alpha}\right)$, always in absorption (see right panel Fig. 1). We normalized the spectra to a continuum of unity because this allows a much better assessment of the true extension of the line wings. Finally we subtracted them to obtain the true emission from the envelope $W^{\text {env }}\left(\mathrm{H}_{\alpha}\right)$ corrected for photospheric absorption:

$W^{\mathrm{env}}\left(\mathrm{H}_{\alpha}\right)=W^{\mathrm{spc}}\left(\mathrm{H}_{\alpha}\right)-W^{\mathrm{mdl}}\left(\mathrm{H}_{\alpha}\right)$.

This value is quoted in Table 5 for LMC and SMC stars and in Table 8 for MWG stars.

As explained before, the spectral type range of the targets ranges from $\mathrm{B} 0$ to $\mathrm{B} 1$. The corresponding equivalent widths cover a range of $\Delta\left(E W_{\text {model }}\right) \simeq 0.1 \AA$ for the same spectral types (Kurucz 1979). Added quadratically to the observational spectroscopic errors, this results in a final uncertainty of less than $1.5 \AA$ except for the MWG source XTE J1946+274, which is $2.0 \AA$.

In Fig. 2 we display $E^{\text {total }}(B-V)$ vs. $W^{\text {env }}\left(\mathrm{H}_{\alpha}\right)$ for all our programme stars. Milky Way binaries are represented by (red) squares, LMC binaries by (blue) asterisks and SMC binaries by (green) triangles. Several conclusions can be drawn from inspection of the plot:

1. total excesses for Milky Way Galaxy stars are of the order of 10 times higher than for stars in the Magellanic Clouds; 
M. S. Riquelme et al.: Circumstellar emission in Be/X-ray binaries of the Magellanic Clouds and the Milky Way

Table 4. Spectral type in the Morgan-Keenan system and its corresponding parameters $T_{\text {eff }},(B-V)_{0}, M_{V}$ and $\log g$, taken from Schmidt \& Kaler (1982).

\begin{tabular}{|c|c|c|c|c|c|c|}
\hline Star & MK & $T(K)$ & $(B-V)_{0}$ & $M_{V}$ & $\log g$ & $W^{\mathrm{mdl}}\left(\mathrm{H}_{\alpha}\right)$ \\
\hline CAL E & $\mathrm{B} 0 \mathrm{~V}^{a}$ & 30000 & -0.30 & -4.0 & 4.0 & 2.7 \\
\hline CAL 9 & $\mathrm{~B} 0 \mathrm{~V}^{a}$ & 30000 & -0.30 & -4.0 & 4.0 & 2.7 \\
\hline RX J0516.0-6916 & $\mathrm{B} 0.5 \mathrm{IV}^{b}$ & 27000 & -0.28 & -4.2 & 3.5 & 2.4 \\
\hline RX J0520.5-6932 & $09 \mathrm{~V}^{a}$ & 33000 & -0.31 & -4.5 & 4.0 & 2.0 \\
\hline RX J0529.8-6556 & $\mathrm{B} 0.5 \mathrm{~V}^{a}$ & 28000 & -0.28 & -3.5 & 4.0 & 3.0 \\
\hline EXO 0531.1-6609 & $\mathrm{B} 0.7 \mathrm{~V}^{a}$ & 27000 & -0.28 & -3.4 & 4.0 & 3.2 \\
\hline RX J0531.5-6518 & $\mathrm{B} 2 \mathrm{~V}^{a}$ & 22000 & -0.24 & -2.4 & 4.0 & 4.2 \\
\hline RX J0535.0-6700 & $\mathrm{B} 0 \mathrm{~V}^{a}$ & 30000 & -0.30 & -4.0 & 4.0 & 2.7 \\
\hline 1A $0538-66$ & $\mathrm{~B} 0.5 \mathrm{~V}^{a}$ & 28000 & -0.28 & -3.5 & 4.0 & 3.0 \\
\hline SAX J0544.1-7100 & $\mathrm{B} 0 \mathrm{~V}^{c}$ & 30000 & -0.30 & -4.0 & 4.0 & 2.7 \\
\hline Н 0544-66 & $\mathrm{B} 0 \mathrm{~V}^{a}$ & 30000 & -0.30 & -4.0 & 4.0 & 2.7 \\
\hline RX J0051.9-7311 & $\mathrm{B} 9.5 \mathrm{~V}^{d}$ & 31000 & -0.31 & -4.3 & 4.0 & 2.5 \\
\hline RX J0052.1-7319 & $\mathrm{B} 0 \mathrm{~V}^{d}$ & 30000 & -0.30 & -4.0 & 4.0 & 2.7 \\
\hline RX J0059.2-7138 & $\mathrm{B} 1 \mathrm{III}^{d}$ & 24000 & -0.27 & -4.4 & 3.5 & 2.8 \\
\hline RX J0101.0-7206 & $\mathrm{B} 1 \mathrm{~V}^{d}$ & 25000 & -0.27 & -3.2 & 4.0 & 3.6 \\
\hline RX J0101.3-7211 & $\mathrm{B} 0.5 \mathrm{~V}^{d}$ & 28000 & -0.28 & -3.5 & 4.0 & 3.0 \\
\hline 4U 0115+634 & $\mathrm{B} 0.2 \mathrm{~V}^{e}$ & 29000 & -0.29 & -3.8 & 4.0 & 2.8 \\
\hline 3A $0726-260$ & $08.5 \mathrm{~V}^{f}$ & 34000 & -0.32 & -4.7 & 4.0 & 1.9 \\
\hline GRO J1008-57 & $\mathrm{B} 0 \mathrm{III} / \mathrm{V}^{e}$ & $29000 / 30000$ & -0.30 & $-5.1 /-4.0$ & $3.5 / 4.0$ & $2.0 / 2.7$ \\
\hline LS 1698 & $\mathrm{~B} 0 \mathrm{III} / \mathrm{V}^{g}$ & $29000 / 30000$ & -0.30 & $-5.1 /-4.0$ & $3.5 / 4.0$ & $2.0 / 2.7$ \\
\hline 1A $1118-61$ & O9.5 $\mathrm{V}^{h}$ & 31000 & -0.31 & -4.3 & 4.0 & 2.5 \\
\hline GX 304-1 & $\mathrm{B} 0.7 \mathrm{~V}^{i}$ & 27000 & -0.28 & -3.4 & 4.0 & 3.2 \\
\hline SS 397 & $\mathrm{~B} 1 \mathrm{~V}^{m}$ & 25000 & -0.27 & -3.2 & 4.0 & 3.6 \\
\hline XTE J1946+274 & B $0-1 \mathrm{~V}^{j}$ & $30000 / 25000$ & $-0.30 /-0.27$ & $-4.0 /-3.2$ & 4.0 & $2.7 / 3.6$ \\
\hline KS $1947+300$ & $\mathrm{~B} 0 \mathrm{~V}^{k}$ & 30000 & -0.30 & -4.0 & 4.0 & 2.7 \\
\hline Cep X-4 & $\mathrm{B} 1-2 \mathrm{~V}^{l}$ & $25000 / 22000$ & $-0.27 /-0.24$ & $-3.2 /-2.4$ & 4.0 & $3.6 / 4.2$ \\
\hline $4 \mathrm{U} 2206+54$ & $09.5 \mathrm{~V}^{m}$ & 31000 & -0.31 & -4.3 & 4.0 & 2.5 \\
\hline SAX J2239.3+6116 & $\mathrm{B} 0 \mathrm{~V}^{i}$ & 30000 & -0.30 & -4.0 & 4.0 & 2.7 \\
\hline
\end{tabular}

Notes. ${ }^{(a)}$ Negueruela \& Coe (2002), ${ }^{(b)}$ Liu et al. (2000), ${ }^{(c)}$ Coe et al. (2001), ${ }^{(d)}$ Mc Bride et al. (2008), ${ }^{(e)}$ Negueruela \& Okazaki (2001), ${ }^{(f)}$ Negueruela et al. (1996), ${ }^{(g)}$ Reig \& Roche (1999), ${ }^{(h)}$ Janot-Pacheco et al. (1981), ${ }^{(i)}$ Ziolkowski (2002), ${ }^{(j)}$ Wilson et al. (2003), ${ }^{(k)}$ Negueruela et al. (2003), ${ }^{(l)}$ Bonnet-Bidaud \& Mouchet (1998), ${ }^{(m)}$ Status as BeX still unknown.

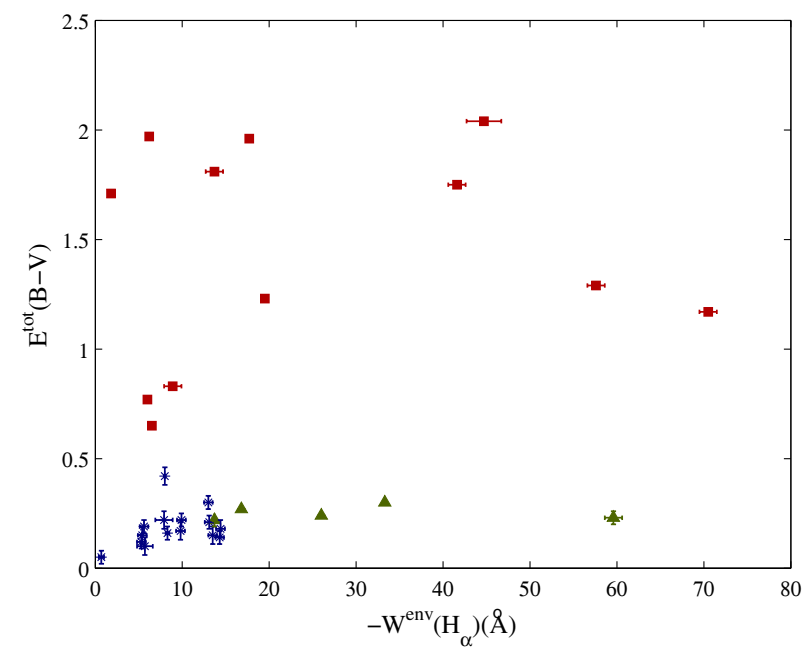

Fig. 2. Relationship between $E^{\text {total }}(B-V)$ and the $W^{\text {env }}\left(\mathrm{H}_{\alpha}\right)$ for the Milky Way (red squares), LMC (blue asterisks) and SMC (green triangles) programme stars. Some stars have error bars of the order of the the symbol size.

2. the LMC and SMC stars occupy a narrow band in this plot, indicating a relationship. No apparent relation is observed for the MWG stars;

3. the SMC sources tend to have much stronger emissions than the LMC sources.
Point 1 can be explained by the MWG Be/X-ray binaries location on the Galactic Plane, where the amount of interstellar material is large, while LMC and SMC sources are observed at high galactic latitudes and suffer from very low interstellar extinction.

Since the $E^{\mathrm{is}}(B-V)$ is essentially constant for the LMC and SMC sources (but see below), points 1 and 2 strongly suggest that the lack of correlation for the MWG stars is entirely attributable to the interstellar reddening term. The relationship between $E^{\mathrm{cs}}(B-V)$ and the amount of emission for MWG stars that should otherwise be apparent, is smeared out because $E^{\text {is }}(B-V)$ is totally independent for each star. In view of this, we will henceforth assume that the relation observed for LMC and SMC stars also holds for the MWG.

Point 3 is surprising. Indeed, there are no strong $\mathrm{H}_{\alpha}$ emitters within our LMC sample while there is only one mild emitter within our SMC sample. A dichotomy seems to exist around $-W\left(\mathrm{H}_{\alpha}\right) \approx 15 \AA$. The MWG, however, displays the whole range of emissions. This could be caused by an observational bias owing to low number statistics. In order to explore this point further we also plotted the non simultaneous photometric and spectroscopic observations of Coe et al. (2005, open triangles) and Antoniou et al. (2009, open circles) in Fig. 3, left panel. Evidently, they occupy the same locus on average, although with a larger scatter. Few mild emitters seem to be present in the Coe et al. (2005) and Antoniou et al. (2009) samples. But because the number of sources is still very small, we dit not proceed with this question here. 

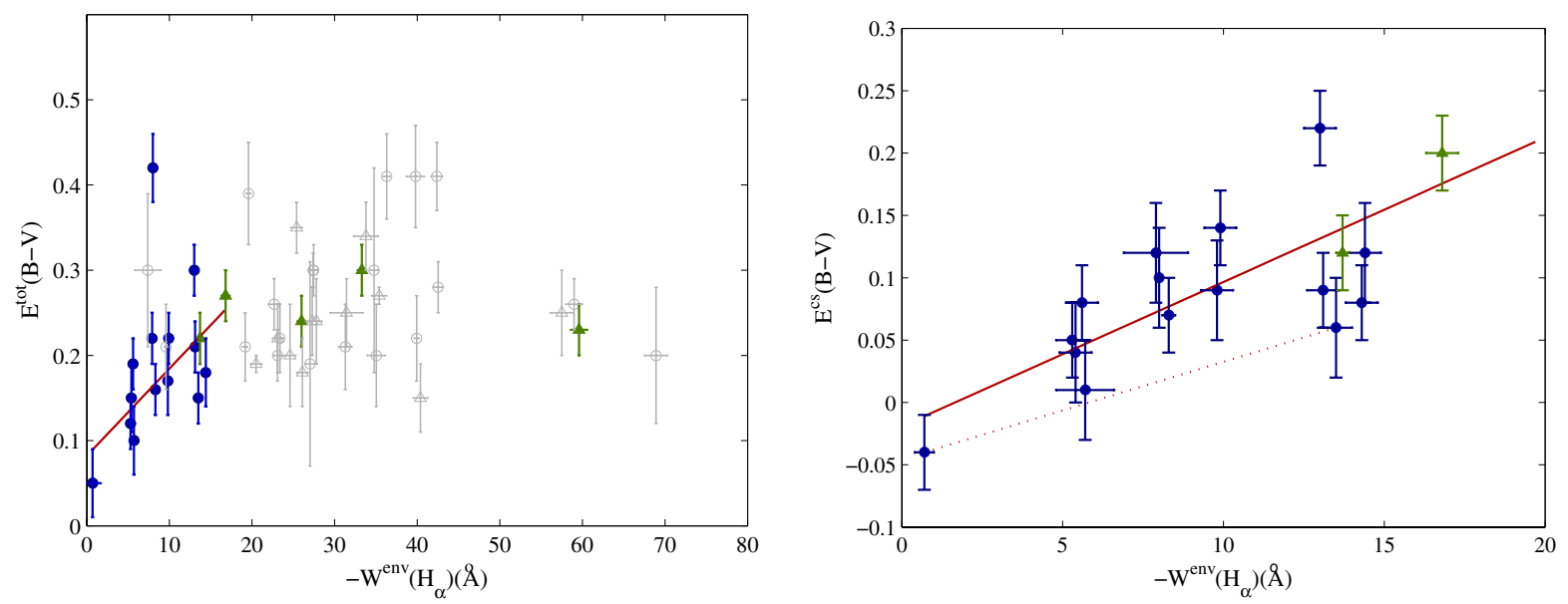

Fig. 3. Left panel: $E^{\text {total }}(B-V)$ vs. $W^{\text {env }}\left(\mathrm{H}_{\alpha}\right)$, for LMC (blue circles) SMC (green triangles). Open triangles belong to SMC stars data from Coe et al. (2005) and spectral types from McBride et al. (2008). Open circles are data from Antoniou et al. (2009). The best fit is plotted for all stars with envelope emission lowerer than $15 \AA$. Right panel: $E^{\mathrm{cs}}(B-V)$ vs. $W^{\text {env }}\left(\mathrm{H}_{\alpha}\right)$ for stars with emission lower than $15 \AA$. Symbols as in left panel. The bottom line links two points that correspond to two different observations of 1A 0538-66.

\section{3. $E^{c s}(B-V)$ vs. $W^{e n v}\left(H_{\alpha}\right)$}

If we represent only stars in the Clouds, (see Fig. 3, left panel), it is possible to appreciate some degree of correlation between both parameters for emission strengths $-W^{\text {env }}\left(\mathrm{H}_{\alpha}\right)$ below $\sim 15 \AA$. Beyond this point, however, the relationship saturates. Then, as a first approximation, we performed a least-squares fit for stars with emission weaker than $15 \AA$ and obtained

$$
\begin{aligned}
E^{\mathrm{total}}(B-V)=-(0.011 \pm 0.003) W^{\mathrm{env}}\left(\mathrm{H}_{\alpha}\right)+(0.08 & \pm 0.03) \\
r & =0.67,
\end{aligned}
$$

where $r$ represents the correlation coefficient. The zero point, $0.08 \pm 0.02 \mathrm{mag}$, would correspond to the total excess without any emission at all, that is to say, the interstellar excess. This is, indeed, the average reddening of the Clouds if one takes the corresponding values in Table 5. This is encouraging. One of the stars from the LMC appears displaced upwards from the rest. This star, SAX J0544.1-7100, is in the southern part of the LMC where the interstellar reddening is higher (it is well known that a gradient exists in the LMC interstellar excess that increases towards the south, while the reddening across the SMC face seems to be more uniform). Indeed, according to Cusumano (1998), this source seems to have an even greater excess which would reach 0.32 mag.

Consequently, we subtracted the individual $E^{\text {is }}(B-V)$ for each star. This excess has two contributions. On one hand there is the foreground interstellar reddening towards the Clouds. For this purpose we used the reddening maps of Schwering \& Israel (1991). On the other hand there is the interstellar reddening within the Clouds. In this case we used the surveys performed by Zaritsky et al. (2004) for the LMC and Zaritsky et al. (2002) for the SMC. In this manner, we calculated the individual circumstellar excess for each star by means of $E^{\text {cs }}(B-V)=$ $E^{\text {total }}(B-V)-E^{\text {is }}(B-V)$. Surprisingly with this approach, some of the resulting circumstellar excesses turned out to be negative, which means that we were over-correcting for reddening. Furthermore, the scatter in the relationship increases. As stated previously, the independent term in Eq. (7) is already very close to the interstellar value to the LMC. There are several reasons why introducing the intracloud reddening does not improve the correlation. As stated by Zaritsky et al. (2002, 2004), there are a number of caveats when using the estimated extinctions. For example, the extinction values are only reliable if a number of stars are used to compute the average value. However, for the positions of our sources there is always a very high dispersion around the average value, sometimes $\sim 100 \%$. Furthermore there seem to be effects due to the position of an object along the line-of-sight. All these effects contribute to increase the scatter in the correlations. Notwithstanding, the slope of the obtained correlations was similar within the errors. Finally, we used also the more recent and accurate reddening maps of the Clouds by Haschke et al. (2011). These maps have been obtained with two independent methods involving the red clump in colour magnitude diagrams and the absolute magnitudes of RR Lyrae stars, respectively. The obtained reddenings are less than $0.03 \mathrm{mag}$ for the vast majority of our sources, much lower than the foreground value and well within the photometric accuracy of our measurements. Unfortunately, not all our sources are covered by the Haschke et al. (2011) survey. Therefore, we applied only the foreground correction. We present the $E^{\mathrm{cs}}(B-V)$ vs. $W^{\text {env }}\left(\mathrm{H}_{\alpha}\right)$ in Fig. 3, right panel. Obviously, the scatter of points is reduced and the degree of correlation is improved. We now obtain the following relation:

$$
\begin{array}{r}
E^{\mathrm{cs}}(B-V)=-(0.011 \pm 0.003) W^{\mathrm{env}}\left(\mathrm{H}_{\alpha}\right) \\
r=0.7 .
\end{array}
$$

The zero point in this equation should be strictly null, since both parameters are pure envelope contributions. Indeed, this is less than the photometric errors and, therefore, compatible with zero. On the other hand, the slope of the relation remains almost unchanged, indicating that subtraction of individual reddenings does not introduce any artificial trend.

Finally, although there are a few points with $E W\left(\mathrm{H}_{\alpha}\right) \geq$ $-15 \AA$, it is quite obvious that a saturation occurs around $0.17 \pm$ 0.03 mag. The non contemporaneous data from Coe et al. (2005) and Antoniou et al. (2009) also support this conclusion. In Fig. 3, left panel, there are several stars with total excesses well above the mean. The majority of those stars indeed present intracloud reddening values of the order of $\sim 0.23 \mathrm{mag}$. In these data sets the photometry and the spectroscopy are not simultaneous. Therefore we will use below only stars within our sample. Taking into account the above discussion, we propose as the 
M. S. Riquelme et al.: Circumstellar emission in Be/X-ray binaries of the Magellanic Clouds and the Milky Way

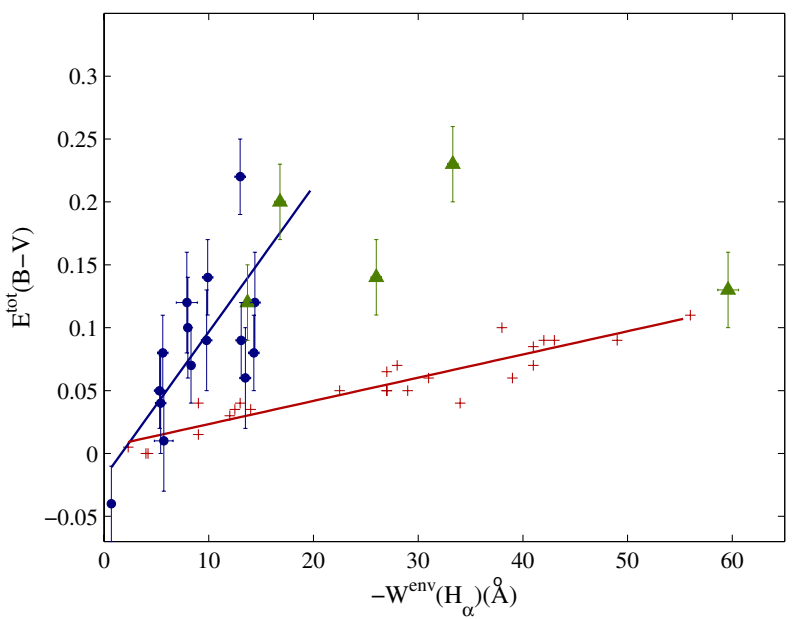

Fig. 4. Correlation for Be/X-ray binaries (filled blue dots) compared with the similar correlation for isolated Be stars (red crosses).

definitive calibration the following equations:

$E^{\mathrm{cs}}(B-V)=\left\{\begin{array}{cl}-(0.011 \pm 0.003) W^{\mathrm{env}}\left(\mathrm{H}_{\alpha}\right) & \text { if }-W^{\text {env }}\left(\mathrm{H}_{\alpha}\right)<15 \AA \\ \sim 0.17 \pm 0.03 & \text { if }-W^{\text {env }}\left(\mathrm{H}_{\alpha}\right) \geq 15 \AA .\end{array}\right.$

Comparing the first of these last equations with that valid for the isolated Be stars

$E^{\mathrm{cs}}(B-V)=-0.002 W\left(\mathrm{H}_{\alpha}\right)$

given by Dachs et al. (1988) (Eq. (39) of that reference), we see that the amount of circumstellar reddening in $E^{\mathrm{cs}}(B-V)$ is about five times higher for Be stars in binaries with the same amount of emission ${ }^{3}$, that is to say, the same value of $W\left(\mathrm{H}_{\alpha}\right)$.

In the extensive study performed by Dachs et al. (1988) on a sample of isolated Be stars, the presence of saturation can also be seen in all infrared colours (Figs. 1 to 5 in that reference). However, this is not observed in the optical colour (Fig. 10 in that reference). The circumstellar excess in isolated Be stars, however, is always less than 0.1 mag even for the stronger emissions, while it is much higher for BeX stars (see Fig. 4). This lack of saturation in the excess at optical wavelengths is also confirmed by Fabregat \& Torrejón (1998) using Strömgren photometry of (isolated) Be stars in open clusters (Fig. 3 of that reference). Therefore, there must exist a fundamental difference in the physical properties of circumstellar envelopes of Be stars in $\mathrm{Be} / \mathrm{X}$-ray binaries with respect to their isolated counterparts because the saturation is present here at higher photon energies.

Finally, 1A 0538-66 was observed twice in different runs. In Fig. 5 we present the spectra of this source in two different states. During the second run the source showed an $\mathrm{H}_{\alpha}$ line in absorption with an equivalent width consistent with the expected photospheric value, that is to say, the Be star was without envelope. The corresponding points are connected by a dotted line in Fig. 3. As can be seen a) it correctly sets the zero point of the calibration (within the photometric errors) and b) it follows the slope of the calibration almost exactly. This behaviour is encouraging and suggests that the calibration will be giving the corresponding value for each individual star and not only the statistical trend.

\footnotetext{
3 Although these authors used equivalent widths not corrected for photospheric absorption.
}

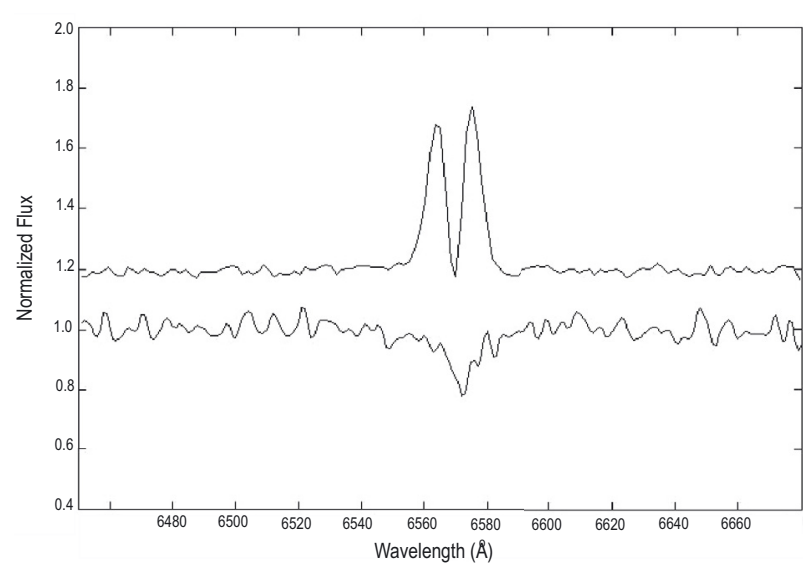

Fig. 5. $\mathrm{H}_{\alpha}$ line shown by $1 \mathrm{~A} 0538-66$ in two different epochs.

$$
\text { 3.4. } E^{\text {total }}(V-I) \text { vs. } W^{e n v}\left(H_{\alpha}\right)
$$

As stated before, the nature of the circumstellar excess is such that it increases with wavelength. For heavily obscured systems, it is often useful to use the infrared colour excess because it diminishes with increasing wavelength. One of the indices that we can obtain from our sample is $(R-I)$. However, the $\mathrm{H}_{\alpha}$ line is located within the wavelength range of the $R$ broadband filter. This results in contaminated values of $R$, rendering its use as a measure of the slope of the continuum very problematic. In view of this, we instead explored the relationship between $(V-I)$ and the amount of emission.

We applied the same procedure as described above for $(B-$ $V)$ to this near-infrared colour $(V-I)$. The total excess is now defined as

$E^{\text {total }}(V-I)=(V-I)_{\mathrm{obs}}-(V-I)_{0}$,

where the intrinsic colour $(V-I)_{0}$ is obtained from Ducati (2001) (listed here in Table 4$)$ and $(V-I)_{\text {obs }}$ is obtained from our photometry (Table 1). In Fig. 6 we display the $(V-I)$ total excesses, calculated in this way (Table 6), for all programme stars with measured $I$ magnitude. Unfortunately, we were able to use only four stars from the LMC sample and none from the SMC (because no $I$ is available). For the SMC we therefore added data from Coe et al. (2005). Using only LMC stars, we obtain the following equation:

$E^{\mathrm{total}}(V-I)=-(0.040 \pm 0.005) W^{\mathrm{env}}\left(\mathrm{H}_{\alpha}\right)+(0.19 \pm 0.04)$.

Stars with the higher levels of emission, $-W^{\text {env }}\left(\mathrm{H}_{\alpha}\right)>15 \AA$, clearly present a saturation around $0.60 \pm 0.10 \mathrm{mag}$.

This can now be compared with the analogous relation for $(B-V)($ Eq. (8)). Evidently the infrared correlation presents a higher slope (about four times higher), as expected. This is consistent with the fact that the circumstellar excess increases towards the red. This statement is based on a very small number of data points and it is only suggestive.

Again, the zero point of the relation should be the total excess for zero emission, that is to say, the interstellar reddening towards the LMC. From Eq. (12) this turns out to be $E^{\text {is }}(V-I)=0.19 \pm 0.04$.

In a totally independent way, we can also obtain this value using the relation between interstellar excesses at different wavelengths given by Fitzpatrick (1999),

$E_{\mathrm{LMC}}^{\mathrm{is}}(V-I)=1.6 E_{\mathrm{LMC}}^{\mathrm{is}}(B-V)$, 
Table 5. Total, interstellar and circumstellar excesses for Magellanic Cloud programme stars as well as their $\mathrm{H}_{\alpha}$ emissions and photosphere corrected $\mathrm{H}_{\alpha}$ emissions.

\begin{tabular}{llcccccc}
\hline \hline & & $E^{\text {total }}(B-V)$ & $E^{\text {is }}(B-V)$ & $E^{\text {cs }}(B-V)$ & $W^{\text {spc }}\left(\mathrm{H}_{\alpha}\right)$ & $W^{\text {env }}\left(\mathrm{H}_{\alpha}\right)$ & $\pm \delta\left(W^{\text {env }}\left(\mathrm{H}_{\alpha}\right)\right)$ \\
\hline LMC & CAL E & $0.16 \pm 0.03$ & 0.09 & $0.07 \pm 0.03$ & -5.6 & -8.3 & 0.2 \\
& CAL 9 & $0.21 \pm 0.03$ & 0.12 & $0.09 \pm 0.03$ & -10.4 & -13.1 & 0.5 \\
& RX J0516.0-6916 & $0.15 \pm 0.04$ & 0.11 & $0.04 \pm 0.04$ & -3.0 & $-5.4^{* *}$ & 0.5 \\
& RX J0516.0-6916 & $0.19 \pm 0.03$ & 0.11 & $0.08 \pm 0.03$ & -3.2 & $-5.6^{* *}$ & 0.5 \\
& RX J0520.5-6932 & $0.30 \pm 0.03$ & 0.08 & $0.22 \pm 0.03$ & -11.0 & -13.0 & 0.5 \\
& RX J0529.8-6556 & $0.12 \pm 0.03$ & 0.07 & $0.05 \pm 0.03$ & -2.3 & -5.3 & 0.5 \\
& EXO 0531.1-6609 & $0.17 \pm 0.04$ & 0.08 & $0.09 \pm 0.04$ & -6.6 & -9.8 & 0.5 \\
& EXO 0531.1-6609 & $0.22 \pm 0.03$ & 0.08 & $0.14 \pm 0.03$ & -6.7 & -9.9 & 0.5 \\
& RX J0531.5-6518 & $0.18 \pm 0.04$ & 0.06 & $0.12 \pm 0.04$ & -10.2 & -14.4 & 0.5 \\
& RX J0531.5-6518 & $0.14 \pm 0.03$ & 0.06 & $0.08 \pm 0.03$ & -10.1 & -14.3 & 0.5 \\
& RX J0535.0-6700 & $0.22 \pm 0.04$ & 0.10 & $0.12 \pm 0.04$ & -5.2 & -7.9 & 1.0 \\
& 1A 0538-66 & $0.05 \pm 0.03$ & 0.09 & $0.00 * \pm 0.03$ & 2.3 & -0.7 & 0.3 \\
& 1A 0538-66 & $0.15 \pm 0.04$ & 0.09 & $0.06 \pm 0.04$ & -10.5 & $-13.5 *$ & 0.5 \\
& SAX J0544.1-7100 & $0.42 \pm 0.04$ & 0.32 & $0.10 \pm 0.04$ & -5.3 & -8.0 & 0.1 \\
& H 0544-66 & $0.10 \pm 0.04$ & 0.09 & $0.01 \pm 0.04$ & -3.0 & -5.7 & 0.9 \\
SMC J0051.9-7311 & $0.28 \pm 0.03$ & 0.07 & $0.21 \pm 0.03$ & -14.6 & -17.1 & 0.5 \\
& RX J0052.1-7319 & $0.30 \pm 0.03$ & 0.07 & $0.23 \pm 0.03$ & -30.6 & -33.3 & 0.5 \\
& RX J0059.2-7138 & $0.24 \pm 0.03$ & 0.10 & $0.14 \pm 0.03$ & -23.2 & -26.0 & 0.5 \\
& RX J0101.0-7206 & $0.23 \pm 0.03$ & 0.10 & $0.13 \pm 0.03$ & -56.0 & -59.6 & 1.0 \\
& RX J0101.3-7211 & $0.22 \pm 0.03$ & 0.10 & $0.12 \pm 0.03$ & -10.7 & -13.7 & 0.2 \\
\hline
\end{tabular}

Notes. ${ }^{(*)}$ In this particular case (lack of emission), $E^{\text {is }}(B-V)$ is greater than $E^{\text {total }}(B-V)$, so that $E^{\text {cs }}(B-V)=-0.04$ becomes negative. This is unphysical. It simply reflects the photometric uncertainty in the zero point. In consequence, we set this point to zero. ${ }^{\left({ }^{* *}\right)}$ Shell line.

along with the corresponding values of $E^{\text {is }}(B-V)$ from Table 5. The average value of $E(V-I)$ derived from Eq. (13) turns out to be $0.13 \pm 0.06$, which is compatible with the previously obtained value of $0.19 \pm 0.04$ within the errors.

Taking into account the previous discussion and a mean insterstellar excess of 0.13 for the SMC (obtained by applying Eq. (13) to the $E^{\text {is }}(B-V)$ of SMC stars taken from the Schwering $\&$ Israel 1991, reddening maps), we propose the following equation:

$E^{\mathrm{cs}}(V-I)=\left\{\begin{array}{cl}-(0.040 \pm 0.005) W^{\mathrm{env}}\left(\mathrm{H}_{\alpha}\right) & \text { if }-W^{\mathrm{env}}\left(\mathrm{H}_{\alpha}\right)<15 \AA \\ \sim 0.47 \pm 0.12 & \text { if }-W^{\mathrm{env}}\left(\mathrm{H}_{\alpha}\right) \geq 15 \AA\end{array}\right.$

where only data from our sample were used. Again, since this is based on a very small number of data points, the result is only suggestive.

The values of $E^{\mathrm{cs}}(V-I)$ and $E^{\text {is }}(V-I)$ so obtained are shown in Table 6 . These equations are based on a quite small and heterogeneous sample though. The values of the calculated circumstellar excesses in $(V-I)$ must be taken, therefore, with due caution.

Finally we note that the total excesses of MWG and LMC stars displayed in Fig. 6 are closer than those displayed in Fig. 2 for $(B-V)$. This can be understood if we consider that total excesses in $(V-I)$ will now have a higher contribution from the circumstellar component compared to the interstellar component (compare Eqs. (8) and (12)).

\section{4. $V$ absorption and emission}

Once $E^{\text {is }}(B-V)$ is known, we can estimate the $V$ extinction by using $A_{V}=3.1 E^{\text {is }}(B-V)$. This expression assumes a standard reddening law, which will not always hold (Fitzpatrick 1999). But it will almost exactly match the low reddenings of the Clouds. The value thus calculated, however, cannot be used to compute the distance modulus by direct comparison with the
Table 6. $(V-I)$ excesses for LMC and Milky Way stars for those stars with photometry in $I$ filter.

\begin{tabular}{lccc}
\hline \hline Star & $E^{\text {tot }}(V-I)$ & $E^{\text {cs }}(V-I)$ & $E^{\text {is }}(V-I)$ \\
\hline LMC & & & \\
CAL E & 0.51 & 0.33 & 0.18 \\
CAL 9 & $\ldots$ & 0.52 & $\ldots$ \\
RX J0520.5-6932 & 0.69 & 0.52 & 0.17 \\
RX J0529.8-6556 & 0.46 & 0.21 & 0.25 \\
1A 0538-66 & 0.18 & 0.03 & 0.15 \\
& & & \\
MWG & & & \\
& & & \\
SS 397 & 2.23 & 0.50 & 1.73 \\
XTE 1946+274 & $2.55 / 2.53$ & 0.50 & $2.05 / 1.03$ \\
KS 1947+300 & 1.74 & 0.78 & 0.96 \\
X Cep X-4 & $1.86 / 1.84$ & 0.50 & $1.36 / 1.34$ \\
4U 2206+54 & 0.73 & 0.26 & 0.47 \\
SAX J2239.3+6116 & 2.25 & 0.55 & 1.70 \\
4U 0115+634 & $\ldots$ & 0.07 & $\ldots$ \\
3A 0726-260 & 1.20 & 0.36 & 0.84 \\
GRO J1008-57 & 2.84 & 0.71 & 2.13 \\
LS 1698 & 1.10 & 0.27 & 0.83 \\
1A 1118-61 & 1.85 & 0.50 & 1.35 \\
GX 304-1 & 2.70 & 0.25 & 2.45 \\
\hline
\end{tabular}

Notes. The uncertainty is less than 0.06 mag for total excess and 0.10 mag for circumstellar and interstellar excesses.

absolute magnitude for the corresponding spectral type of the star. The reason, of course, is that the $V$ magnitude is also affected by the envelope. The classical Be stars brighten with increasing emission, that is to say, they are brighter when redder (Zorec \& Briot 1991; Fabregat \& Torrejón 1998). However, shell Be stars ${ }^{4}$ show the opposite trend (Harmanec 1983). Two stars in

4 These are believed to be Be stars viewed edge-on so that the circumstellar envelope self-absorbs the circumstellar emission. 
M. S. Riquelme et al.: Circumstellar emission in Be/X-ray binaries of the Magellanic Clouds and the Milky Way

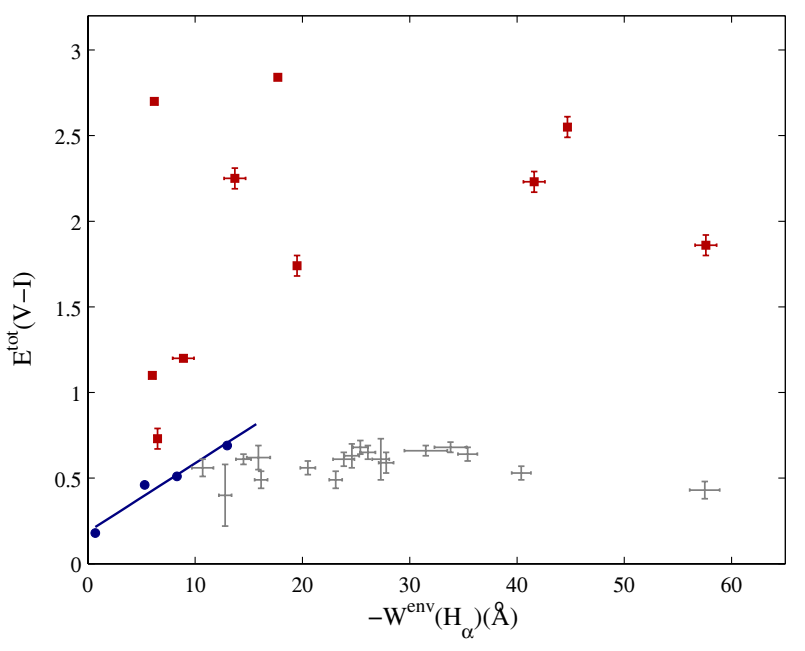

Fig. 6. $E^{\text {tot }}(V-I)$ vs. $W^{\text {env }}\left(\mathrm{H}_{\alpha}\right)$, for LMC (squares), Milky Way stars (triangles). Crosses belong to the SMC data of Coe et al. (2005) and spectral types from McBride et al. (2008). The line represents the relationship for LMC stars only. Some stars have error bars of the order of the the symbol size.

our LMC sample have shown the shell phenomenon in the spectrum $^{5}$ in the past, RXJ 0516.0-6916 and 1A0538-66. We will restrict ourselves here to the classical Be stars and therefore will not consider these two stars in the following discussion, even though they presented non-shell spectra during our campaign.

In principle, we could follow the same approach as for the colour excesses. We know the total extinction in $V$ by means of $A_{V}^{\text {tot }}=V_{\text {obs }}-V_{0}=V-V_{0}$, where $V_{0}$ can be derived from the known luminosity class and distance to the LMC and SMC. The net effect over the $V$ magnitude is a combination of two contributions:

$A_{V}^{\text {total }}=A_{V}^{\text {is }}+E_{V}^{\mathrm{cs}}$.

The two terms in Eq. (15) have opposite signs, positive for interstellar absorption and negative for the circumstellar emission term. The final brightness of the star depends on each particular combination of interstellar extinction and circumstellar brightening. Since the interstellar term is known a priori we can isolate the $E_{V}^{\mathrm{cs}}$ and compare it with the known values of $-W^{\mathrm{env}}\left(\mathrm{H}_{\alpha}\right)$, in the same way as we did with the $(B-V)$ excess. Unfortunately, this procedure does not give any observable trend within our programme stars in the Clouds.

In view of this, we will explore whether the value given by Dachs et al. (1988) (Eq. (40) in that reference) for isolated Be stars is valid for our objects. The circumstellar emission in $V$ is related to the emission-line strength through the equation

$E_{V}^{\mathrm{cs}} \approx-0.1\left(\frac{W^{\mathrm{env}}\left(\mathrm{H}_{\alpha}\right)}{-30 \AA}\right)$,

which, according to these authors, accounts for the excess emission from a circumstellar envelope caused by free-free and freebound transitions.

In order to find a suitable relationship for Be/X-ray binaries, we considered a relationship of the form

$E_{V}^{\mathrm{cs}}=R_{\mathrm{cs}} E^{\mathrm{cs}}(B-V)$.

\footnotetext{
${ }^{5}$ Central absorption in the $\mathrm{H} \alpha$ emission line below the continuum level.
}

Replacing $E^{\mathrm{cs}}(B-V)$ by Eq. (10) and $E_{V}^{\mathrm{cs}}$ by Eq. (16), we obtain $R_{\mathrm{cs}}=1.7$ for isolated Be stars.

Assuming now that this factor also holds for Be/X-ray binaries, we would have

$E_{V}^{\mathrm{cs}}=1.7\left(-0.011 W^{\mathrm{env}}\left(\mathrm{H}_{\alpha}\right)\right)$,

where we made use of our calibration (Eq. (9)). As stated in the introduction, and in agreement with the results presented in previous sections, there is evidence that the physical properties of circumstellar envelopes in Be/X-ray binaries differ significantly from those of isolated Be stars. Therefore the previous assumption might not hold. However, as will be shown below in Sect. 5, only the above relation yields the correct values for the independently known distances to our sources, both in the Clouds and the MWG.

For the sake of comparison, we recast the previous equation in the form

$E_{V}^{\mathrm{cs}} \approx-0.6\left(\frac{W^{\mathrm{env}}\left(\mathrm{H}_{\alpha}\right)}{-30 \AA}\right)$.

Comparing this equation with Eq. (16) we see that the excess in $V$ would be six times higher for Be stars in binaries.

On the other hand, for stars with $-W^{\text {env }}\left(\mathrm{H}_{\alpha}\right)>15 \AA$, the saturation regime holds and

$E^{\mathrm{cs}}(V)=-1.7 \cdot 0.17=-0.3$,

independent of the amount of emission.

Therefore, we propose the following calibration, valid for $\mathrm{Be} / \mathrm{X}$-ray binaries, under the assumption that the factor $R_{\mathrm{cs}}$ is the same for isolated $\mathrm{Be}$ stars and $\mathrm{Be} / \mathrm{X}$-ray binaries:

$A_{V}^{\text {tot }}= \begin{cases}3.1 E^{\text {is }}(B-V)-0.6\left(\frac{W^{\text {env }}\left(\mathrm{H}_{\alpha}\right)}{-30 \AA}\right) & \text { if }-W^{\text {env }}\left(\mathrm{H}_{\alpha}\right)<15 \AA \\ 3.1 E^{\text {is }}(B-V)-0.3 & \text { if }-W^{\text {env }}\left(\mathrm{H}_{\alpha}\right) \geq 15 \AA .\end{cases}$

\section{Distance modulus}

Next we evaluate the goodness (self-consistency) of the correction proposed in the preceding section by computing the distance to the Magellanic Clouds, using our programme stars, with three different methods:

1. The method commonly found in the literature, using the total excess $A_{V}=3.1 E^{\text {total }}(B-V)$. This method implicitly (and certainly incorrectly) assumes that the total-to-selective absorption in the $V$ band has exactly the same expression (and sign) for the interstellar and circumstellar terms.

2. We compute the distance using only the correctly calculated interstellar value $A_{V}=3.1 E^{\text {is }}(B-V)$. This method would completely neglect the brightening of the Be star by the circumstellar emission.

3. Finally, we use the $V$ excess determined by mean of Eq. (21), which correctly takes into account both effects, but assumes that the $E^{\mathrm{cs}}(B-V)$ contribution to total emission valid for isolated Be stars also holds for Be/X-ray binary stars.

Now, for each one of these methods, we computed the distance modulus with the commonly used expression:

$D M=V-M_{V}-A_{V}$,

where $V$ is the observed value taken from Table $1, M_{V}$ is the absolute magnitude for the corresponding spectral type and luminosity classs and $A_{V}$ as proposed in the preceding section. The 
Table 7. Distance modulus for Magellanic Clouds stars calculated with the three methods explained in the text.

\begin{tabular}{lcccc}
\hline \hline Galaxy & Star & DM1 & DM2 & DM3 \\
\hline LMC & CAL E & 17.67 & 17.96 & 18.12 \\
& CAL 9 & 18.16 & 18.61 & 18.87 \\
& RX J0520.5-6932 & 18.00 & 18.44 & 18.73 \\
& RX J0529.8-6556 & 17.92 & 18.10 & 18.20 \\
& EXO 0531.1-6609 & 17.72 & 18.06 & 18.25 \\
& EXO 0531.1-6609 & 17.54 & 17.88 & 18.07 \\
& RX J0531.5-6518 & 17.93 & 18.42 & 18.71 \\
& RX J0531.5-6518 & 18.06 & 18.54 & 18.83 \\
& RX J0535.0-6700 & 18.26 & 18.53 & 18.69 \\
& SAX J0544.1-7100 & 17.93 & 18.20 & 18.36 \\
\hline mean & & 18.0 & 18.3 & 18.5 \\
$\sigma$ & & 0.2 & 0.3 & 0.3 \\
\hline SMC & RX J0051.9-7311 & 17.82 & 18.44 & 18.65 \\
& RX J0052.1-7319 & 17.93 & 18.55 & 18.76 \\
& RX J0059.2-7138 & 17.73 & 18.35 & 18.55 \\
& RX J0101.0-7206 & 18.21 & 18.83 & 19.03 \\
& RX J0101.3-7211 & 18.55 & 19.02 & 19.29 \\
\hline mean & & 18.1 & 18.6 & 18.9 \\
$\sigma$ & & 0.3 & 0.3 & 0.3 \\
\hline
\end{tabular}

resulting individual values, mean values and standard deviation for LMC and SMC are quoted in Table 7, where DM1 corresponds to the first method, DM2 to the second and DM3 to the third. Obviously $D M 1$ differs significantly from the other methods, while DM2 and DM3 are compatible within the errors.

The next step was to compare the results obtained by all three methods with the distance to the Clouds obtained independently with dedicated surveys. Storm et al. (2004) obtained $18.48 \pm 0.07$ for LMC and $18.88 \pm 0.13$ for SMC based on metallicity-dependent Cepheids calibrations. These values excellently agree with those derived through other independent methods such as large samples of eclipsing binaries, which deliver $D M=18.91 \pm 0.03 \pm 0.1$ for the SMC (Hilditch et al. 2005).

There are two important caveats. On one hand these are $a v$ erage distances. Distance determinations from several eclipsing binaries in the LMC suggest that there is a considerable depth along the line of sight (Fitzpatrick et al. 2003). The SMC is also known to be very extended along the line of sight, with many estimates well in excess of $10 \mathrm{kpc}$ (Mathewson et al. 1986; Crowl et al. 2001). In a recent survey Subramaniam \& Subramaniam (2009) found that, indeed, the SMC has a greater depth than the LMC. More important, these authors have pointed out the possible existence of a bulge in the optical center of the SMC where both the extinction and the depth are larger than in outer areas. Within our SMC sample, two stars show DM larger than the average value of the Cloud. These are located in the outer areas. The other three, in turn, show $D M$ values lower than the Cloud average. These are located near the optical centre of the Cloud. This can be understood if we consider the increased optical depth of the bulge, which would prevent us from observing sources behind it, much in the same way as the MWG bulge does. In turn, the sources located far from the optical centre of the Cloud can be observed at greater distances. This possible explanation is based on a very small number of sources, however, and is, therefore, very tentative.

These effects can explain the scatter of $\sigma=0.3 \mathrm{mag}$ obtained within our small sample and why the direct determination of the $V$ excess, described at the beginning of the previous section, does not work. On the other hand, the luminosity classes of many stars in our sample have been determined without taking into account the possible brightening of the Be star caused by the circumstellar emission. That is to say, a star classified as class III could actually be class V. Only two class III stars are included in our sample, one in the SMC and one in the LMC. The difference in absolute magnitude from luminosity class III to class $\mathrm{V}$ for the spectral types concerned here is $\Delta M_{V} \simeq 0.4$. This is compatible with the standard deviation given in Table 7.

The proposed correction was based on the assumption that the ratio of circumstellar emission in $V$ with respect to $(B-V)$ is $R_{\mathrm{cs}}=1.7$, obtained from isolated Be stars. In order to check the validity of this assumption, we varied this factor in steps of 0.05 and recalculated the distance to the Clouds. Any departure from the assumed value resulted in a significant worsening of the computed distance. Furthermore, as will be shown in the next section, only this factor yields the consistent results for Milky Way sources whose distances have been computed by completely independent methods.

As can be seen in Table 7, Method 1 (the one commonly used) gives completely inaccurate values. The second and third methods significantly improve the distance to the Clouds and they are compatible within the errors. However, it is only the third one which delivers the correct mean value. Therefore we propose method three as the the best one and we will use it to compute the distance to MWG binaries in the next section.

\section{Milky Way stars}

Even though the extrapolation to the MWG of calibrations based on stars in other galaxies might not be entirely accurate, we will assume in the following that such an assumption is tenable. The fact that no Be/X-ray binaries are known to exist in any MWG open cluster leaves, however, little margin to assume otherwise if we want to move forward in this matter. In any case, this is a fundamental limitation that affects any calibration based on stars in the Clouds. It has been shown that the lower metallicity of the SMC probably has implications on its higher abundance of Be stars (Martayan et al. 2010) and on the onset of the Be phenomenon. However, once the Be star is formed, there seem to be no fundamental differences between Be stars in the SMC, the LMC and the MWG.

Therefore we applied Eqs. (9) and 21 to the MWG sources of our sample. First we computed the circumstellar excesses in $(B-V)$ from the $W^{\text {env }}\left(\mathrm{H}_{\alpha}\right)$ from Eq. (9), as explained in previous sections. Then we subtracted this excess from the total and obtained the interstellar excess through Eq. (2).

This excess, along with the photosphere-corrected circumstellar emission, allows to estimate $A_{V}^{\text {total }}$ by means of Eq. (21). Finally we computed the distance through the commonly used equation:

$d=10^{\left(V-M_{V}-A_{V}^{\mathrm{tot}}+5\right) / 5}$.

In Table 8 we present the results for the MWG programme stars. For the shell stars no distances were computed because Eqs. (21) are not applicable. For the stars with a remaining uncertainty on their spectral type, both possible distances are given.

We now compare the computed distances with previous determinations. In Table 9 we show the distances obtained with the proposed calibration to those compiled from the literature.

Evidently, our distances exceed those previously published. Throughout explicitly excluding the brightening of the source caused by circumstellar emission leads to a underestimation of the distance. In two cases, our distance determinations are much higher than those quoted in Raguzova \& Popov (2005). In both 
M. S. Riquelme et al.: Circumstellar emission in Be/X-ray binaries of the Magellanic Clouds and the Milky Way

Table 8. Parameters obtained for Milky Way stars. Shell stars have neither $A_{V}$ nor distance because they do not satisfy Eq. (19).

\begin{tabular}{lccccccc}
\hline \hline Star & $E^{\text {total }}(B-V) \pm 0.03$ & $W^{\text {spc }}\left(\mathrm{H}_{\alpha}\right)$ & $W^{\text {env }}\left(\mathrm{H}_{\alpha}\right)$ & $E^{\mathrm{cs}}(B-V)$ & $E^{\text {is }}(B-V)$ & $A_{V}^{\text {total }} \pm 0.31$ & $d(\mathrm{kpc})$ \\
\hline 4U 0115+63 & 1.71 & 1.0 & $-1.8 \pm 0.1$ & $0.02 \pm 0.01$ & 1.69 & 5.2 & $7.0 \pm 0.3$ \\
3A 0726-260 & 0.83 & -7.0 & $-8.9 \pm 1.0$ & $0.10 \pm 0.03$ & $0.73 \pm 0.04$ & 2.1 & $7.0 \pm 0.5$ \\
GRO J1008-57 & 1.96 & -15.0 & $-17.0 /-17.7 \pm 0.5$ & $0.17 \pm 0.04$ & $1.79 \pm 0.05$ & 5.2 & $9.7 \pm 0.8 / 5.8 \pm 0.5$ \\
LS 1698 & 0.77 & $-4.0^{*}$ & $-6.0 /-6.7 \pm 0.2^{*}$ & $0.07 \pm 0.01$ & 0.70 & $\ldots$ & $\ldots$ \\
1A 1118-61 & 1.17 & -68.0 & $-70.5 \pm 1.0$ & $0.17 \pm 0.05$ & $1.00 \pm 0.06$ & 2.8 & $5.2 \pm 0.9$ \\
GX 304-1 & 1.97 & $-3.0^{*}$ & $-6.2 \pm 0.2^{*}$ & $0.07 \pm 0.01$ & $1.90 \pm 0.03$ & $\ldots$ & 4.6 \\
SS 397 & 1.75 & -38.0 & $-41.6 \pm 1.0$ & $0.17 \pm 0.05$ & $1.58 \pm 0.06$ & $1.5 \pm 0.2$ \\
XTE J1946+274 & $2.04 / 2.01$ & -42.0 & $-44.7 /-45.6 \pm 2.0$ & $0.17 \pm 0.05$ & $1.87 / 1.84 \pm 0.06$ & $5.5 / 5.4$ & $12.0 \pm 1.7 / 8.7 \pm 1.2$ \\
KS 1947+300 & 1.23 & -16.8 & $-19.5 \pm 0.5$ & $0.17 \pm 0.04$ & $1.06 \pm 0.05$ & 3.0 & $10.4 \pm 0.9$ \\
Cep X-4 & $1.29 / 1.26$ & -54.0 & $-57.6 /-58.2 \pm 1.0$ & $0.17 \pm 0.05$ & $1.12 / 1.09 \pm 0.06$ & $3.2 / 3.1$ & $7.9 \pm 1.2 / 5.9 \pm 0.9$ \\
4U 2206+54 & 0.65 & -4.0 & $-6.5 \pm 0.5$ & $0.07 \pm 0.02$ & $0.58 \pm 0.04$ & 1.7 & $2.9 \pm 0.2$ \\
SAX J2239.3+6116 & 1.81 & -11.0 & $-13.7 \pm 1.0$ & $0.15 \pm 0.04$ & $1.66 \pm 0.05$ & 4.9 & $5.4 \pm 0.5$ \\
\hline
\end{tabular}

Notes. ${ }^{(*)}$ Shell line.

Table 9. Distances for MWG stars calculated using our proposed method compared to those previously published in the literature.

\begin{tabular}{lccc}
\hline \hline Source & $\begin{array}{c}d \text { (this work) } \\
(\mathrm{kpc})\end{array}$ & $\begin{array}{c}d \text { (previous) } \\
(\mathrm{kpc})\end{array}$ & Ref. \\
\hline KS1947+300 & $10.4 \pm 0.9$ & 10 & RP05 \\
4U2206+54 & $2.9 \pm 0.2$ & 2.5 & RP05 \\
SAXJ2239.3+61 & $5.4 \pm 0.5$ & 4.4 & RP05 \\
3A0726-26 & $7.0 \pm 0.5$ & 6.0 & RP05 \\
1A1118-61 & $5.2 \pm 0.9$ & 5.0 & RP05 \\
Cep X-4 & $7.9 \pm 1.2 / 5.9 \pm 0.9$ & 3.8 & RP05 \\
GROJ1008-57 & $9.7 \pm 0.8 / 5.8 \pm 0.5$ & 2.0 & RP05 \\
XTE 1946+27 & $12.0 \pm 1.7 / 8.7 \pm 1.2$ & $9.5 \pm 2.9$ & Wi03 \\
4U0115+63 & $7.0 \pm 0.3$ & $7-8$ & NO01 \\
\hline
\end{tabular}

Notes. RP05 (Raguzova \& Popov 2005). Wi03 (Wilson et al. 2003). NO01 (Negueruela \& Okazaki 2001).

cases, though, the discrepancy comes from the assumed parameters and has nothing to do with the method presented here. For example GRO J1008-57: the distance quoted in Raguzova \& Popov (2005) was taken from a work by Haberl \& Sasaki (2005). These last authors used, in turn, results from Macomb et al. (1994). These authors estimated a $V=13.9 \pm 0.2$ mag for the counterpart using a cross calibration of archive plates. Our more accurate observed value is $V=15.08 \pm 0.02$, which accounts for the difference. Our derived distance, however, agrees well with the estimate made by Petre \& Gehrels (1994) for luminosity class V. Remarkably, this distance puts the source right on the outer Sagittarius arm and, together with the argument by Petre \& Gehrels (1994), would argue in favour of a mainsequence counterpart.

The case of $4 \mathrm{U} 0115+63$ is a fortunate one because this system was observed by Negueruela \& Okazaki (2001) in a diskless state. These authors favour a $7 \mathrm{kpc}$ distance and warn that this could be an upper limit since there could be still some residual emission present. Considering that we observed the source with significant $\mathrm{H}_{\alpha}$ emission, the agreement is excellent, and constitutes an independent check of the validity of this work.

Likewise, the distance to XTE J1946+27 has been estimated using completely independent methods based on other energy ranges. Wilson et al. (2003) estimated a distance of $9.5 \pm 2.9 \mathrm{kpc}$ based on a correlation between measured spin-up rate and X-ray flux. This value excellently agrees with ours.

Figure 7 shows the distribution of our sources on the Galactic Plane. As can be seen, and as is expected, the binaries trace the

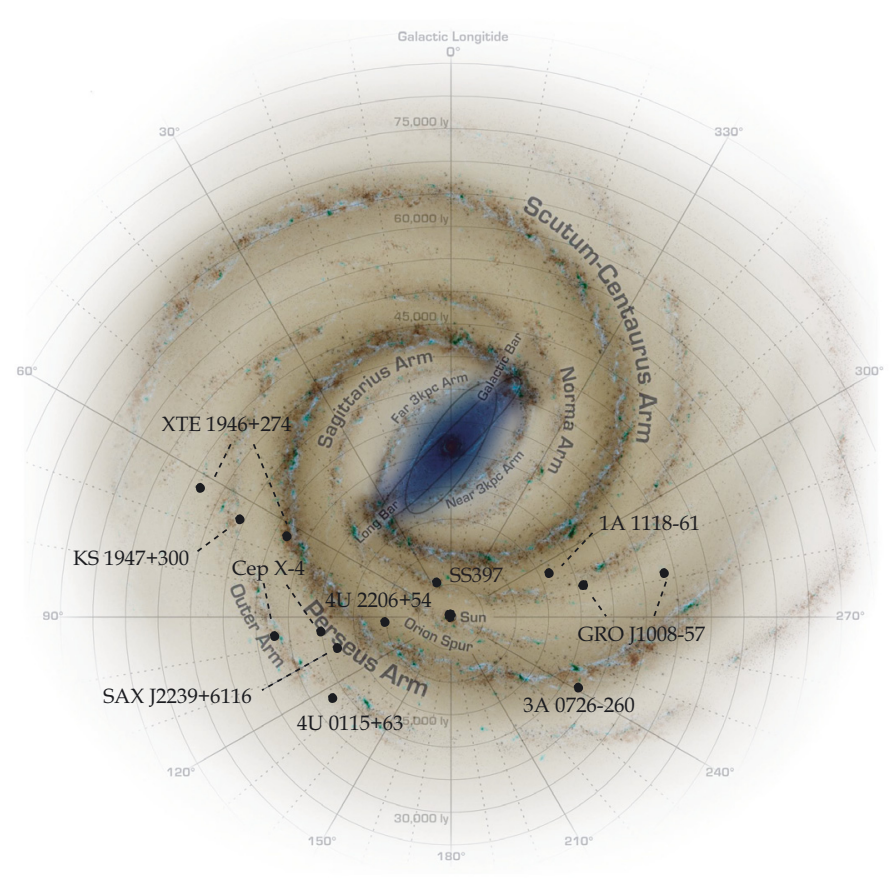

Fig. 7. Sketch of the Milky Way (based on NASA Spitzer satellite data) showing the position of the Be/X-ray binaries of our sample. Sources with two possible spectral types are connected by a line.

arms of the MWG, following the location of the OB star populations. Sources such as SS397, GRO J1008-57 (as V class) and 1A 1118-61 belong to the outer Sagittarius Arm. The group of SAX J2239.3+61, 4U 2206+54, 3A 0726-26, GRO J1008-57 (as III class), Cep X-4 (as B2) y XTE J1946+274 (as B1), trace the Perseus arm. Finally, XTE J1946+274 (as B0), $\mathrm{KS} 1947+300, \mathrm{Cep} \mathrm{X}-4$ (as B1) and 4U 0115+63, trace the outer Norma arm.

\section{Summary and discussion}

We summarize here the main conclusions and equations:

- The total reddening in the Milky Way sources is always much higher than for Magellanic Cloud sources, an effect attributable to the interstellar component. 
- The circumstellar contribution to the $(B-V)$ colour can be computed by means of the equation

$$
E^{\mathrm{cs}}(B-V)=\left\{\begin{array}{cl}
-0.011 W^{\mathrm{env}}\left(\mathrm{H}_{\alpha}\right) & \text { if }-\mathrm{W}^{\mathrm{env}}\left(\mathrm{H}_{\alpha}\right)<15 \AA \\
\sim 0.17 & \text { if }-\mathrm{W}^{\mathrm{env}}\left(\mathrm{H}_{\alpha}\right)>15 \AA .
\end{array}\right.
$$

- This result confirms that $E^{\mathrm{cs}}(B-V)$ is about five times higher for Be stars in binaries than for isolated Be stars with the same amount of emission ( same $-W^{\text {env }}\left(\mathrm{H}_{\alpha}\right)$ ). As we pointed out in the introduction, the circumstellar envelopes of Be stars in binaries are geometrically smaller and optically thicker than the envelopes in isolated Be stars. Therefore it is plausible that the electron density in the envelopes of the former is higher than in the latter. This has been pointed out by Zamanov et al. (2001). The circumstellar continuum emission in turn will also be higher in the former because the Bremsstrahlung emission coefficient $j_{v} \sim \gamma n_{\mathrm{e}} n_{\mathrm{i}}$ is proporcional to the electron density, as is the the circumstellar reddening.

- The study of the near-infrared colour $(V-I)$ suggested a similar behaviour albeit with a steeper slope and saturation level. This agrees with the fact that circumstellar reddening increases towards the red (see for example Dachs et al. 1988). This conclusion is based on a very small number of data points and therefore must be regarded as tentative.

- In order to compute the distance to a Be/X-ray binary (non shell Be star) in the MWG, we must include the circumstellar contribution to the observed $V$ magnitude by means of

$$
A_{V}^{\text {total }}=\left\{\begin{array}{cl}
3.1 E^{\text {is }}(B-V)-0.6\left(\frac{W^{\text {env }}\left(\mathrm{H}_{\alpha}\right)}{-30 \AA}\right) & \text { if }-W^{\text {env }}\left(\mathrm{H}_{\alpha}\right)<15 \AA \\
3.1 E^{\text {is }}(B-V)-0.3 & \text { if }-W^{\text {env }}\left(\mathrm{H}_{\alpha}\right) \geq 15 \AA .
\end{array}\right.
$$

The first term is the commonly used correction for interstellar absorption while the second one takes into account the over luminosity of Be stars. Neglecting the increase in $V$ brightness caused by circumstellar emission results in an underestimation of the distance (hence luminosity) for the $\mathrm{Be} / \mathrm{X}$-ray binaries. The importance of this effect depends on the combination of interstellar extinction and circumstellar emission for a particular source. It shows that the maximum value for the overluminosity for $\mathrm{BeX}$ with strong $\mathrm{H}_{\alpha}$ emission is $0.3 \mathrm{mag}$. This last relationship was obtained assuming that the ratio $R_{\mathrm{cs}}=E^{\mathrm{cs}}(V) / E^{\mathrm{cs}}(B-V)=1.7$ deduced from isolated $\mathrm{Be}$ stars also holds for $\mathrm{Be} / \mathrm{X}$-ray binaries. However, this assumption seems to be justified because any departure from this ratio yields unacceptable values of the independently known distances to BeX stars in the Magellanic Clouds and the Milky Way Galaxy.

\section{Appendix A: Distance errors}

We calculate the errors in the distance for Milky Way stars, $\sigma_{d}$, showed in Table 8, using error propagation. We start with

$d=10^{\left(V-M_{V}-A_{V}+5\right) / 5}$.

Then we differentiate Eq. (A.1)

$\frac{\sigma_{d}}{d}=\left(\frac{\sigma_{V}}{5}+\frac{\sigma_{\mathrm{A}_{\mathrm{V}}^{\text {total }}}}{5}\right) \ln 10$,

and solve for $\sigma_{d}$,

$\sigma_{d}=\frac{d}{5}\left(\sigma_{V}+\sigma_{A_{V}^{\text {total }}}\right) \ln 10$, where $\sigma_{V}$ is taken from Table 2 and $\sigma_{A_{V}^{\text {total }}}$ comes from Eqs. (21) for non-saturated stars by means of

$\sigma_{A_{V}^{\text {total }}}($ nosat $)=3.1 \sigma\left(E^{\text {is }}(B-V)\right)+\frac{0.6}{30} \sigma\left(W^{\text {env }}\left(\mathrm{H}_{\alpha}\right)\right)$,

while for saturated stars the errors are

$\sigma_{A_{V}^{\text {total }}}($ sat $)=3.1 \sigma\left(E^{\text {is }}(B-V)\right)$.

In Eq. (A.4), the first term is

$\sigma\left(E^{\mathrm{is}}(B-V)\right)=\sqrt{\left(\sigma\left(E^{\mathrm{total}}(B-V)\right)\right)^{2}+\left(\sigma\left(E^{\mathrm{cs}}(B-V)\right)\right)^{2}}$,

where $\sigma\left(E^{\text {total }}(B-V)\right)$ is taken from Table 2 and $\sigma\left(E^{\mathrm{cs}}(B-V)\right)$ comes from Eq. (9) in this way:

$\sigma\left(E^{\mathrm{cs}}(B-V)\right)=0.011 \sigma\left(W^{\mathrm{env}}\left(\mathrm{H}_{\alpha}\right)\right)+0.0017 W^{\mathrm{env}}\left(\mathrm{H}_{\alpha}\right)$.

The first term in this equation takes into account the error when measuring $W^{\text {env }}\left(\mathrm{H}_{\alpha}\right)$ in the spectrum, listed in Table 8 . The second term corresponds to the error in the slope of the circumstellar excess calibration (Eq. (9)).

Acknowledgements. This research has been partially supported by the Spanish Ministerio de Ciencia e Innovación, MICINN, under grant AYA2010-15431. The authors gratefully acknowledge the anonymous referee, whose comments and suggestions improved the content of the paper.

\section{References}

Antoniou, V., Hatzidimitriou, D., Zezas, A., \& Reig P. 2009, ApJ, 707, 1080 Bonnet-Bidaud, J. M., \& Mouchet, M. 1998, A\&A, 332, L9

Coe, M. J., Negueruela, I., Buckley, D. A. H., Haigh, N. J., \& Laycock, S. G. T. 2001, MNRAS, 324, 623

Coe, M. J., Edge, W. R. T., Galache, J. L., \& McBride, V. A. 2005, MNRAS, 356,502

Crowl, H. H., Sarajedini, A., Piatti, A. E., et al. 2001, AJ, 122, 220

Cusumano, G., Israel, G. L., Mannucci, F., et al. 1998, A\&A, 337, 772

Dachs, J., Engels, D., \& Kiehling, R. 1988, A\&A, 194, 167

Draper, P. W., \& Gray, N. 2000, Starlink User Note 214.7, R.A.L.

Draper, P. W., Taylor, M., \& Allan, A. 2000, Starlink User Note 139.12, R.A.L.

Ducati, J. R., Bevilacqua, C. M., Rembold, S. B., \& Ribeiro, D. 2001, ApJ, 558, 309

Fabregat, J., \& Torrejón, J. M. 1998, A\&A 332, 643

Fernández Barba D. 2005, Ph.D. Thesis, University of Barcelona, Dpto. Astronomy \& Meteorology, Area de conocimiento: ciencias experimentales y matematicas

Fitzpatrick, E. L. 1999, PASP, 111, 63

Fitzpatrick, E. L., Ribas, I., Guinan, E. F., et al. 2003, ApJ, 587, 685

Gies, D. R., Bagnuolo, W. G., Baines, E. K., et al. 2007, ApJ, 654, 527

Grundstrom, E. D., \& Gies, D. R. 2006, ApJ, 651, L53

Harmanec, P. 1983, Hvar Obs. Bull., 7, 55

Haschke, R., Grebel, E. K., \& Duffau, S. 2011, AJ, 141, 158

Hilditch, R. W., Howarth, I. D., \& Harries, T. J. 2005, MNRAS, 357, 304

Howarth, I., Murray, J., Mills, D., \& Berry, D. S. 1998, Starlink User Note 50.21, R.A.L.

Janot-Pacheco, E., Ilovaisky, S. A., \& Chevalier, C. 1981, A\&A, 99, 274

Kurucz, R. L. 1979, ApJS, 40, 1

Landolt, A. U. 1995, VizieR On-line Data Catalog: II/118

Liu, Q. Z., van Paradijs, J., \& van den Heuvel, E. P. J. 2000, A\&AS, 147, 25

Macomb, D. J., Schrader, C. R., \& Schultz, A. B. 1994, ApJ, 437, 845

Martayan, C., Baade, D., \& Fabregat, J. 2010, A\&A, 509, 11

Mathewson, D. S., Ford, V. L., \& Visvanathan, N. 1986, ApJ, 301, 664

McBride, V. A., Coe, M. J., Negueruela, I., Schurch, M. P. E., \& McGowan, K. E. 2008, MNRAS, 388, 1198

Negueruela, I. 1998, A\&A, 338, 505

Negueruela, I., \& Coe, M. J. 2002, A\&A, 385, 517

Negueruela, I., \& Okazaki, A. T. 2001, A\&A, 369, 108

Negueruela, I., \& Reig, P. 2001, A\&A, 371, 1056

Negueruela, I., Roche, P., Buckley, D. A. H., et al. 1996, A\&A, 315, 160

Negueruela, I., Israel, G. L., Marco, A., et al. 2003, A\&A, 397, 739 
M. S. Riquelme et al.: Circumstellar emission in Be/X-ray binaries of the Magellanic Clouds and the Milky Way

Okazaki, A. T. \& Negueruela, I. 2001, A\&A, 337, 161

Petre, R., \& Gehrels, N. 1994, A\&A 282, L33

Raguzova, N. V., \& Popov, S. B. 2005, Astron. Astrophys. Trans., 24, 151

Reig, P. 2011, Ap\&SS 332, 1

Reig, P., \& Roche, P. 1999, MNRAS, 306, 100R

Reig, P., Fabregat, J., \& Coe, M. 1997, A\&A, 322, 193

Schmidt-Kaler, T. H. 1982, Physical parameters of the stars, in LandoltBornstein New Series, Vol. 2b, Astronomy and Astrophysics - stars and star clusters, ed. K. Schaifers, \& H. H. Voigt (New York: Springer, Verlag)

Schwering, P. B. W., \& Israel, F. P. 1991, A\&A, 246, 231

Shortridge, K., Meyerdicks, H., Currie, M., et al. 1997, Stalink User Note 86.15

R.A.L.
Storm, J., Carney, B. W., Gieren, W. P., et al. 2004, A\&A, 415, 531 Subramaniam, S., \& Subramaniam, A. 2009, A\&A, 496, 399S

Torrejón, J. M., Negueruela, I., \& Riquelme, M. S. 2005, in Active OB-stars: Laboratories for Stellar and Circumstellar Physics, Sapporo, Japan, 361, 503 Tycner, C., Gilnreath, G. C., Zavala, R. T., et al. 2006, AJ, 131, 2710

Vilchez, J. M., \& Esteban, C. 1996, MNRAS, 280, 720

Wilson, C. A., Finger, M. H., Coe, M. J., \& Negueruela, I. 2003, ApJ, 584, 996 Zamanov, R. K., Reig, P., Martí, J., et al. 2001, A\&A, 367, 884

Zaritsky, D., Harris, J., Thompson, I. B., et al. 2002, AJ, 123, 855

Zaritsky, D., Harris, J., Thompson, I. B., et al. 2004, AJ, 128, 1606

Ziolkowski, J. 2002, Mem. Soc. Astron. Ital., 73, 1038

Zorec, J., \& Briot, D. 1991, A\&A, 245, 150 